\title{
Biocompatibility, uptake and anti-cancer activity of doxorubicin loaded gold nanoparticles in MCF-7 breast cancer cell line
}

\author{
Bhuvanasree Srinivas Raghavan', Rajaram Anantanarayanan², Rama Rajaram ${ }^{1 *}$ \\ 1 Biochemistry Laboratory, Central Leather Research Institute, Adyar, Chennai, India. \\ 2Bio-Physics Laboratory, Central Leather Research Institute, Adyar, Chennai, India.
}

Received: October 11, 2016; Accepted: October 15, 2016; Published: October 20, 2016

*Corresponding author: Rama Rajaram, Biochemistry Laboratory, Central Leather Research Institute, Adyar, Chennai, India, pin-600 020, Tel: +91 (44) 24437177; Fax: +91 (44) 24911589; E-mail: rajaram.rama@gmail.com

\begin{abstract}
The focus of the present work is on the preparation and loading of the anti-cancer drug doxorubicin (Dox) on the surface of both kaempferol and resveratrol capped gold nanoparticles (k-AuNPs and r-AuNPs) and investigating their cytotoxic properties to MCF-7 cells. The effects of k-AuNP-Dox and r-AuNP-Dox conjugates have been investigated using zebra fish embryos as a model system and the results obtained with respect to hatching and survival rates confirm that they are bio-compatible. Time dependent uptake of these conjugates by MCF-7 cancer cell line has also been evaluated. No significant release of Dox is observed in vitro at neutral $\mathrm{pH}$, whereas at mild acidic $\mathrm{pH}$ environments, drug release is observed from k-AuNP-Dox and r-AuNP-Dox conjugates. The cytotoxic potential of these drug conjugates is better compared to the same concentration of the unconjugated counterparts or free Dox. Reactive oxygen species (ROS) production has been monitored and increased levels are observed within $2 \mathrm{~h}$ of treatment with $20 \mu \mathrm{g} /$ $\mathrm{mL}$ (Au equivalents) of both the nano drug conjugates. Presence of membrane blebs and cleavage of DNA of MCF-7 cells on treatment with k-AuNP-Dox and r-AuNP-Dox conjugates as visualized from the SEM micrographs and agarose gel electrophoresis confirm the mode of cell death to be apoptosis. Cell cycle analysis also indicates G2/M arrest by conjugates which confirm apoptosis. These results suggest that the anti-cancer effects of the bio-compatible nano drug conjugates can be used as a novel therapeutic approach to overcome the toxic side effects of doxorubicin.
\end{abstract}

Keywords: Gold nanoparticles; Doxorubicin; Zebra fish embryo; Cell death; Breast cancer

\section{Introduction}

Doxorubicin (Dox) is an anthracycline antibiotic commonly used as a chemotherapeutic agent for the treatment of several cancers including breast cancer [1]. The main drawbacks of this drug are non-specificity, cardiotoxicity, poor solubility,poor bioavailability and early clearance from the body [2-4]. In order to overcome these demerits and efficiently deliver Dox to tumors, the drug has been loaded to micelles, liposomes, polymers, dendrimers, carbon nanotubes, iron oxide nanoparticles, gold nanorods and gold nanoparticles [5-11].Of these, gold nanoparticles (AuNPs) hold immense promise as a carrier due to its large surface to volume ratio, inertness, easy functionalization, low toxicity and ample cell penetration properties [12, 13]. Conjugations of Dox to AuNPs through covalent linkages and surface adsorption have been attempted [14]. In these methods, AuNPs prepared using chemicals such as sodium borohydride or citrate have been utilized which has resulted in non-compatibility towards normal cells. For overcoming this problem, AuNPs synthesized using plant extracts and pure phytochemicals are suggested as alternatives. In addition, delivery of Dox using these AuNPs to cancer cells is also being investigated in recent times. For example, AuNPs prepared using Helminthosporum solani have been used for Dox delivery to cervical cancer cells (HeLa) [15].Azadirachta indica extract capped AuNPs have also been reported to deliver Dox to embryonic kidney cells (HEK239) [16]. Since k-AuNPs have been shown to elicit toxicity to MCF7 cells and preliminary results obtained in our laboratory have indicated that resveratrol (a stillbenoid) conjugated to AuNPs (r-AuNPs)is also able to bring about apoptosis of MCF-7 cells, it is possible that loading of Dox to k-AuNPs and r-AuNPs may enhance the cytotoxicity [17]. Hence, Dox conjugated k-AuNPs and $r$-AuNPs have been synthesized and characterized and their actions on MCF-7 cells have been investigated.

\section{Experimental Details}

\section{Materials}

Hydrogen tetrachloroaurate $\left(\mathrm{HAuCl}_{4} 3 \mathrm{H}_{2} \mathrm{O},>99.9 \%\right)$, resveratrol, kaempferol, doxorubicin hydrochloride (Dox), resazurin, Dulbecco's Modified Eagles Medium (DMEM), fetal bovine serum (FBS),sodium bicarbonate, Dulbecco'sphosphate buffered saline (PBS), penicillin, streptomycin, amphotericinB, gentamycin, ribonuclease A (RNase), propidium iodide (PI), dic hlorodihydrofluoresceindiacetate (DCFH-DA), 4'-6- diamidino-2- 
phenylindole (DAPI), proteinase K, Tris-HCl, EDTA, NP-40, sodium dodecyl sulphate (SDS), agarose and hexamethyldisilazane (HMS) were purchased from Sigma-Aldrich (USA). Sodium chloride, Sodium hydroxide $(\mathrm{NaOH}>98 \%)$ was obtained from Himedia, India. Thermanox coverslips, sodium cacodylate trihydrate, osmium tetroxide and glutaraldehyde were purchased from Electron Microscopy Sciences, USA. MilliQ water (conductivity of $18 \mathrm{~m} \Omega \mathrm{cm}^{-1}$ ) was autoclaved and filter- sterilized using $0.22 \mu \mathrm{m}$ filters. All chemicals were used as received without any further purification. MCF-7 and HBL-100 cells were obtained from NCCS, Pune.

\section{Preparation of k-AuNPs and r-AuNPs and loading of Dox to AuNPs}

The preparation and characterization of the AuNPs using kaempferol and resveratrol have been performed according to the protocol described previously [17]. Briefly, kaempferol(or resveratrol as the case may be) solutions at $\mathrm{pH} 9$ were added to $\mathrm{HAuCl}_{4}$ solution in an individual reaction vessel at a molar ratio of 2:1(Au: reductant) and kept at constant shaking at room temperature (RT) for the formation of AuNPs. The absorbance of the k-AuNPs and r-AuNPs was recorded using spectrophotometer (Tecan Infinite M200).

Dox loaded AuNPs were prepared according to the method of Mirza et al. [18]. Briefly, Dox (80 $\mu$ g) was added to $15 \mathrm{~mL}$ of $\mathrm{k}$-AuNPs and r-AuNPs in milli $\mathrm{Q}$ water and stirred continuously at RT for $24 \mathrm{~h}$. The solution was protected from light to avoid photodecomposition. After incubation, the sample was centrifuged at 13,000 $\mathrm{xg}$ for $30 \mathrm{~min}$ and washed with water until the supernatant turned colorless. The supernatants were collected and absorbance was read at $490 \mathrm{~nm}$. The amount of Dox bound was calculated using the formula;

$$
\% \text { loading }=\frac{(\text { Drug loaded initially }- \text { free drug in supernatant })}{\text { Drug loaded initially }} \times 100
$$

\section{Characterization of k-AuNPs-Dox and r-AuNPs-Dox conjugates}

Various physico-chemical techniques were used for characterizing the prepared k-AuNPs-Dox and r-AuNPs-Dox conjugates. The hydrodynamic size of the nano drug conjugates was recorded using dynamic light scattering method (DLS) and the surface charge was calculated using zeta potential measurements (Malvern Zetasizer version 6.2). The lyophilized powder was analyzed for the presence of functional groups using Fourier Transform Infrared Spectroscopy (FT-IR) (Nicolet impact spectrophotometer) operating at a resolution of $4 \mathrm{~cm}^{-1}$ in transmission mode from $500-3500 \mathrm{~cm}^{-1}$. The concentration of the gold ions in the conjugates was determined using inductively coupled plasma optical emission spectrometer (ICP-OES).

\section{In Vitro Release Studies}

The release of Dox from k-AuNPs-Dox and r-AuNPs-Dox conjugates was performed according to the method of Aryal etal 2007 [3].The k-AuNPs-Dox and r-AuNPs-Dox conjugates were loaded inside dialysis tubings $(6-8 \mathrm{kDa})$ and suspended in PBS
( $\mathrm{pH}$ of 7.4and later at $\mathrm{pH} 5.8$ ) in a beaker which was placed on a magnetic stirrer with continuous stirring (100 rpm) for $8 \mathrm{~h}$ at $37{ }^{\circ} \mathrm{C}$. In order to measure the drug release, PBS was removed at regular intervals and the absorbance at $490 \mathrm{~nm}$ was measured using a spectrophotometer. Equal volume of PBS was replaced in the beaker. Aliquots were drawn till no further release was noted. The experiment was repeated three times and expressed as mean \pm SD.

\section{In Vivo Biocompatibility study using Zebra fish embryo}

The fertilized eggs of zebra fish (Danio rerio) were collected and segregated using a stereomicroscope before performing the experiments. All the embryos used for the study were derived from the same spawn of eggs for comparison and statistical analysis. The embryos ( 6 per well) were placed in a 24 well plate and each treatment group was present in duplicate wells. Each experiment was repeated two times. The developing larvae were maintained in $30 \%$ Danicau's solution containing $58 \mathrm{mM} \mathrm{NaCl}$, $0.7 \mathrm{mM} \mathrm{KCl}, 0.4 \mathrm{mM} \mathrm{MgSO}_{4}, 5 \mathrm{mM}$, HEPES (pH 7.4) at $30{ }^{\circ} \mathrm{C}$. The fertilized embryos were treated with different concentrations of k-AuNPs-Dox, r-AuNPs-Dox conjugate or free Dox and the embryos were continuously monitored from $4-96$ hours post fertilization (hpf) for the presence of morphological changes or deformities, hatching and survival rates. According to the new EU Directive 2010/63/EU, earlier stages of developing embryos (up to $120 \mathrm{hpf}$ ) do not fall into regulatory framework [19].

\section{Culture of MCF-7 cells.}

Breast cancer cell line (MCF-7)was maintained in DMEM medium supplemented with $10 \% \mathrm{FBS}$ and $1 \%$ antibiotics in a humidified incubator at $37{ }^{\circ} \mathrm{C}$ with $5 \% \mathrm{CO}_{2}$ (Binder, Germany). The cells were passaged every 3-4 days. The cells on reaching confluence were treated with different concentrations (10, 20, 40 and $60 \mu \mathrm{g} / \mathrm{mL}$ of $\mathrm{Au}$ ) of k-AuNPs-Dox and r-AuNPs-Dox conjugate. Free Dox (5 and $10 \mu \mathrm{g} / \mathrm{mL}$ ) corresponding to that present in the 20 and $40 \mu \mathrm{g} / \mathrm{mL}$ of the conjugates was also included for investigations.

\section{Cellular uptake of r-AuNPs-Dox and k-AuNPs-Dox conjugates}

In order to confirm the cellular uptake of the conjugate, fluorescence microscopy and flow cytometry experiments were performed. For confocal laser scanning microscopy (CLSM), cells (MCF-7) were seeded on coverslips, treated with $20 \mu \mathrm{g} / \mathrm{mL}$ of $\mathrm{k}$-AuNPs-Dox and r-AuNPs-Dox and $5 \mu \mathrm{g} / \mathrm{mL}$ of free Dox (dox concentration equivalent to that present in $20 \mu \mathrm{g} / \mathrm{mL}$ of AuNPs conjugate) for 2 and $6 \mathrm{~h}$. Later the coverslips were placed on a glass slide and observed for uptake of Dox using CLSM (Nikon, Japan). For flow cytometry, $1 \times 10^{5}$ cells were seeded in a 12 well plate and allowed to grow overnight. The cells were treated with $20 \mu \mathrm{g} / \mathrm{mL}$ of $\mathrm{k}$ and r-AuNPs alone, k-AuNPs-Dox, r-AuNPs-Dox and free Dox ( $5 \mu \mathrm{g} / \mathrm{mL}$ ) for $2 \mathrm{~h}$ and $6 \mathrm{~h}$. The cells were then trypsinized, washed and the uptake of Dox was analyzed using FACS Calibur (BD Biosciences, USA).

For TEM analysis, the cells after treatment were fixed with glutaraldehyde followed by osmium tetroxide in cacodylate 
buffer ( $\mathrm{pH7.4)}$ for $1 \mathrm{~h}$ and 30 min respectively. Later, dehydration was carried out using series of acetone water mixtures $(25,50$, 75 and $100 \%$ ). This was followed by resin infiltration (Epon resin) with series of resin in acetone $(25,50$ and $75 \%)$ mixtures and finally samples were placed in 'easy molds'with $100 \%$ resin and incubated at $60^{\circ} \mathrm{C}$ overnight. Ultrathin sections of the sample in the resin block was cut (60-80 nm), placed on grid and stained with uranyl acetate and lead citrate solutions. Finally, the sections were viewed under Jeol JEM 1400 TEM at $80 \mathrm{kV}$ and micrographs were captured using Olympus Keen view CCD camera.

\section{Cytotoxic effect of k-AuNPs-Dox and r-AuNPs-Dox conjugate on MCF-7 and HBL-100 cells}

To determine the viability of MCF-7 and HBL-100 cells, resazurin assay has been performed [20].Briefly, cells were washed with PBS and then cells were incubated in fresh medium containing various concentrations of k-AuNPs-Dox, r-AuNPs-Dox $(10 \mu \mathrm{g} / \mathrm{mL}$ to $60 \mu \mathrm{g} / \mathrm{mL})$ and free Dox. Resazurin $(10 \mu \mathrm{g})$ was added and the fluorescence (Ex: $530-590 \mathrm{~nm}$ ) of the supernatants was measured after $6 \mathrm{~h}$. From the viability assay, the $\mathrm{IC}_{50}$ values have been calculated. Viability values were calculated from three independent experiments and expressed as mean \pm SD.

\section{Morphological assessment of MCF-7 cells}

DAPI Staining: Treated cells were washed and fixed in $4 \%$ paraformaldehyde for $30 \mathrm{~min}$. The fixed cells were stained with DAPI (1 $\mu \mathrm{g} / \mathrm{mL}$ ) (Ex: 350 and Em: $470 \mathrm{~nm}$ ) for $20 \mathrm{~min}$ in the dark and observed for nuclear changes using fluorescence microscopy (Euromax, Holland).

Scanning Electron Microscopy (SEM): Cells seeded on sterile Thermanox ${ }^{\circledR}$ coverslips were treated with $20 \mu \mathrm{g} / \mathrm{mL}$ of $\mathrm{k}$-AuNPs-Dox and r-AuNPs-Dox for $24 \mathrm{~h}$. The cells were then fixed with $2 \%$ glutaraldehyde and subsequently with $1 \%$ osmium tetroxide. They were then dehydrated in a series of acetonewater mixtures $(25,50,75$ and $100 \%)$ for 30 min each, followed by dehydration with a series of hexamethyldisilazane (HMS)acetone mixtures (25, 50, 75 and $100 \%)$ for $1 \mathrm{~h}$ each. Finally, cells were lyophilized, placed on stubs with carbon adhesive tapes, sputter coated with gold and observed for morphological changes using SEM (FEI Quanta 200) operating at 30KV.

\section{Cell Cycle Analysis}

MCF-7 cells were treated with 10 and $20 \mu \mathrm{g} / \mathrm{mL}$ of k-AuNPDox and r-AuNP-Dox and $5 \mu \mathrm{g} / \mathrm{mL}$ of free Dox for $24 \mathrm{~h}$. Later the cells were washed with ice cold PBS, fixed with $70 \%$ ethanol and stored at $-20^{\circ} \mathrm{C}$ until analysis. To the fixed cells, ribonuclease A and PI were added, and incubated at $37{ }^{\circ} \mathrm{C}$ for $30 \mathrm{~min}$ in the dark and subsequently analyzed using FACS Calibur flow cytometer (BD Biosciences, USA). The percentage of cells in various phases was analyzed using Cell Quest Pro software. The experiments were repeated three times and expressed as mean \pm SD.

\section{DNA fragmentation}

DNA fragmentation analysis was carried out using $1 \%$ agarose gel electrophoresis. MCF-7 cells were seeded $\left(1 \times 10^{6}\right)$ on 6 well tissue culture plates and exposed to free Dox, Dox conjugated AuNPs for $24 \mathrm{~h}$ and $\mathrm{k}$ or r-AuNPs alone for $48 \mathrm{~h}$. DNA was isolated according to the protocol of Hermann et al [21]. Cells were pelleted, mixed with DNA lysis buffer $(50 \mathrm{mM}$ Tris $\mathrm{HCl}(\mathrm{pH}$ 7.4), $20 \mathrm{mM}$ EDTA and 1\% NP-40) and kept for $30 \mathrm{~min}$ on ice. The lysates were incubated with RNase ( $5 \mathrm{mg} / \mathrm{mL}$ ) and $1 \%$ SDS at $56{ }^{\circ} \mathrm{C}$ for $2 \mathrm{~h}$. This was followed by incubation at $37^{\circ} \mathrm{C}$ for 2 $\mathrm{h}$ with proteinase $\mathrm{K}(2.5 \mathrm{mg} / \mathrm{mL})$. DNA was precipitated with ammonium acetate ( 0.5 volumes) and $70 \%$ of ice cold ethanol (2.5 volumes) at $-80^{\circ} \mathrm{C}$ overnight. The samples were centrifuged at 13,000 x g for $20 \mathrm{~min}$ and DNA was dissolved in Tris-EDTA buffer. The samples were electrophoresed with $1 \%$ agarose gel and the image was documented using gel documentation system (Bio-Rad, USA). DNA fragmentation analysis of HBL-100 cells upon treatment with free Dox and Dox conjugated AuNPs for 24 $\mathrm{h}$ were also performed.

\section{ROS Production}

Cells were seeded $\left(1 \times 10^{5}\right)$ on sterile coverslips and treated with $20 \mu \mathrm{g} / \mathrm{mL}$ of the drug conjugates and after the treatment period, $10 \mu \mathrm{M}$ DCFH-DA probe was added, incubated for $37^{\circ} \mathrm{C}$ and observed for green fluorescence $(488 \mathrm{~nm})$ using fluorescence microscopy. The cells were also analysed for ROS production using FACS Calibur flow cytometer (BD Biosciences, USA).

\section{Statistical Analysis}

All experiments were performed in triplicate and repeated three times. Data were analyzed using two-way analysis of variance followed by Bonferroni post-test (Graph Pad Prism Software Inc., USA version 5.0). The data were considered to be statistically significant at $\mathrm{p}$-value $<0.05$.

\section{Results and Discussion}

\section{Characterization of r-AuNPs-Dox and k-AuNPs-Dox conjugates}

A pure solution of Dox has a characteristic UV-Visible absorption at $490 \mathrm{~nm}$ [22].After adding Dox to k-AuNPs, a shift in the SPR of AuNPs from $535 \mathrm{~nm}$ to $508 \mathrm{~nm}$ and broadening of the peak are observed. Similar changes are observed after addition of Dox tor-AuNPs. This is indicative of binding of Dox on to the AuNPs (Figure.1a and b).This can also be inferred from fluorescence measurements. The fluorescence of Dox (Em: 600 $\mathrm{nm}$ ) is also found to decrease when k-AuNPs or r-AuNPs are added to a solution of Dox (Figure. 1c).Though the relative fluorescence intensity decreases, the wavelength at which emission occurs remains unaltered. This indicates that the structure of Dox remains the same even after getting adsorbed on the surface of AuNPs. Similar quenching of fluorescence intensity of Dox by citrate stabilized AuNPs has been attributed to the transfer of energy from Dox to the AuNPs [23, 24].

In order to eliminate the unbound Dox from the surface of $\mathrm{k}$ and $\mathrm{r}$-AuNPs, successive washing with milli $\mathrm{Q}$ water and centrifugation have been carried out. From the formula mentioned in section 2.2, the Dox loading percentage has been calculated and is observed to be 58 and $60 \%$ for k-AuNP-Dox and $r$-AuNP-Dox respectively. When the pellet is re-suspended 
in milli Q water, the SPR is observed to be sharp with peak at $540 \mathrm{~nm}$ as indicated by the UV-Visible spectra (Figure. 1a and b). The size of k-AuNP-Dox and r-AuNP-Dox as measured using DLS is observed to be $75 \mathrm{~nm}$ and $44 \mathrm{~nm}$ respectively and found to be similar to the unconjugated AuNPs (Table 1). Decrease in the zeta potential values from $-24 \mathrm{mV}$ to $-22 \mathrm{mV}$ and $-33 \mathrm{mV}$ to $-27 \mathrm{mV}$ when the conjugated and the unconjugated k-AuNPs and r-AuNPs are compared, indicates the presence of positively charged amine groups of Dox on the surface of r-AuNPs and k-AuNPs. Zeta potential measured after 5 months for all the samples did not decrease considerably indicating stable AuNPs and AuNP-Dox conjugates (Table 1). A similar decrease in the zeta potential values of citrate capped AuNPs when bound to Dox $(-27 \mathrm{mV}$ to $-21 \mathrm{mV}$ )has been observed and attributed to the inherent positive charge of the Dox [18].In order to find out if physical adsorption of the Dox on to the surface of AuNPs is via electrostatic interaction, $\mathrm{NaCl}$ (an electrolyte solution) of different concentrations were added to k-AuNP-Dox and r-AuNPDox solutions, incubated for $30 \mathrm{~min}$, centrifuged and the Dox fluorescence in the supernatant was recorded(Figure.1d). On increasing the concentration of $\mathrm{NaCl}$ from $0.1 \mathrm{M}$ to $1 \mathrm{M}$, a gradual increase in the fluorescence intensity of the supernatant has been observed which indicates the release of Dox from the AuNPsDox conjugates. Fluorescence of the suspension of the pellets in water shows that removal of Dox from the AuNPs is partial. Similar observations have been made with repeated $\mathrm{NaCl}(0.1-$ $1 \mathrm{M}$ ) washings. On further increasing the concentration of $\mathrm{NaCl}$ to $2 \mathrm{M}$, the fluorescence intensity of the supernatant is found to be high indicating that at this concentration of $\mathrm{NaCl}$, Dox is released efficiently from the AuNPs. Subsequently, after two washes with $2 \mathrm{M} \mathrm{NaCl}$, the pellet when suspended in water does not exhibit any fluorescence indicating that Dox has been released from the AuNPs completely (Figure. 1e). The above observations point to the occurrence of electrostatic interaction of amine groups of Dox with the k-AuNPs and r-AuNPs.

The FT-IR spectrum of free Dox shows peaks at 3420, 1640, $1381 \mathrm{~cm}^{-1}$. The peak at $3420 \mathrm{~cm}^{-1}$ corresponds to the hydroxyl group and peaks at 1640 and $1381 \mathrm{~cm}^{-1}$ corresponds to the N-H stretching of amine group of Dox. In the case of k-AuNP-Dox conjugate, shift in peaks to 3435,1616 and $1389 \mathrm{~cm}^{-1}$ has been observed which is indicative of interaction of the protonated amine group of Dox on the surface of k-AuNPs. The FTIR spectra of r-AuNP-Dox indicate shifts at 3451, $1647 \mathrm{~cm}^{-1}$ with the intensity of the peak at $1396 \mathrm{~cm}^{-1}$ being less indicating the involvement of $\mathrm{N}-\mathrm{H}$ groups and electrostatic binding (Figure. 1f). The involvement of the amine group of Dox in binding to negatively charged citrate or chitosan capped AuNPs has been previously reported $[18,25]$

\section{In vitro drug release}

The drug release profile of Dox loaded k-AuNPs and r-AuNPs have been studied at pH 5.8 and 7.4(Figure.2a \&b). We observed that within $1 \mathrm{~h}, 34.8$ and $12.6 \%$ of Doxis released from r-AuNP-Dox conjugate at $\mathrm{pH} 5.8$ and 7.4 respectively. Around $50 \%$ of the drug at $\mathrm{pH}$ 5.8is released by $6 \mathrm{~h}$ and this remains constant thereafter up to $8 \mathrm{~h}$. On the other hand, at pH 7.4 only $23 \%$ of drug is released after $6 \mathrm{~h}$ and no further release is observed after $8 \mathrm{~h}$. Similarly, in (a)

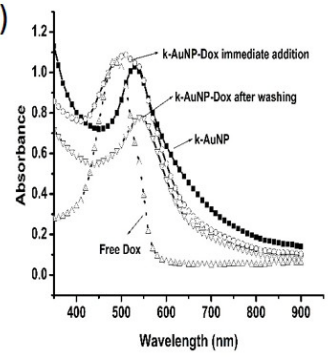

(c)

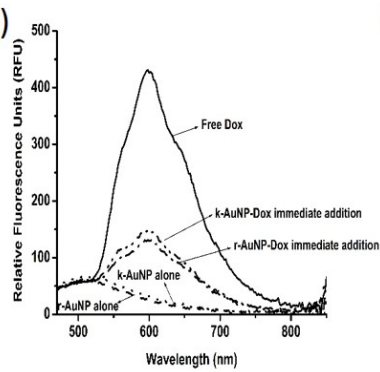

(e)

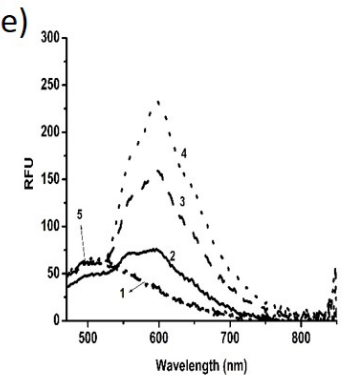

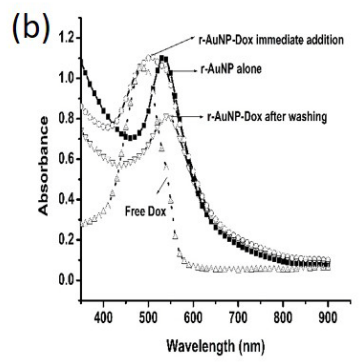

(d)

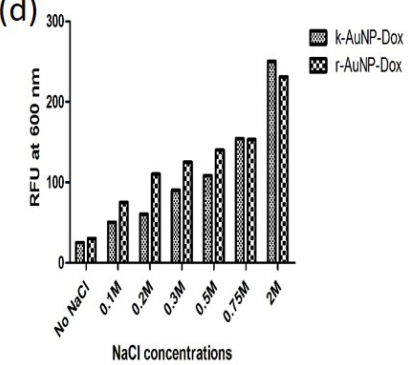

(f)

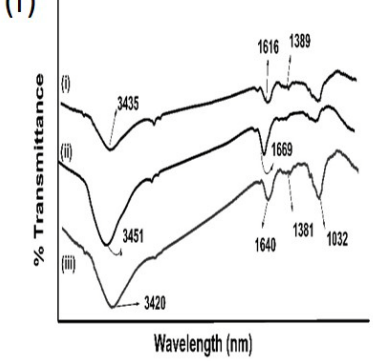

Figure 1: Spectroscopic measurements of AuNP-Dox conjugates. UVVisible spectra of (a) k-AuNP-Dox, (b) r-AuNP-Dox. (c)fluorescence spectra of AuNP alone, AuNP-Dox conjugates and free AuNPs, (d) histogram depicting the fluorescence of Dox in the supernatants obtained after washing the Dox conjugates with different concentrations of $\mathrm{NaCl}$ solutions, (e) fluorescence spectra of Dox present inthe pellets of AuNPDox conjugates obtained after washing with different concentrations of NaCl. (1) NaCl alone, (2) 0.1 M, (3) $0.5 \mathrm{M}$, (4) $2 \mathrm{M}$ after first wash, (5) $2 \mathrm{M}$ after second wash and (f) FT-IR spectra of (i) k-AuNP-Dox, (ii) r-AuNPDox and (iii) free Dox
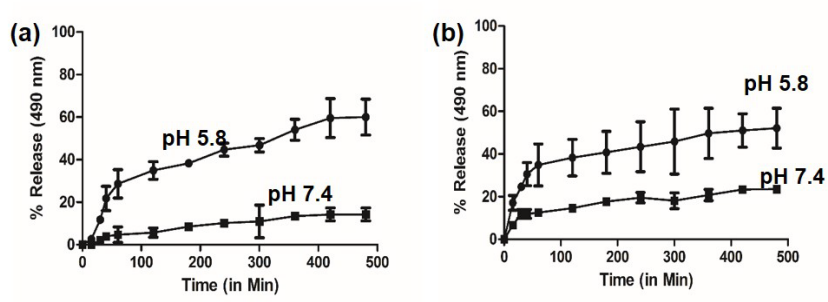

Figure 2: Dox release from (a) k-AuNP-Dox and (b) r-AuNP-Dox at pH 5.8 and 7.4

the case of k-AuNP-Dox, 28.6 and $4.7 \%$ of Dox is released within the first hour at pH 5.8 and 7.4 respectively which reached 54 and $13 \%$ after $6 \mathrm{~h}$. This indicates that the Dox release from the AuNPs is triggered by an acidic environment. It is evident from a previous report that the cytoplasm and endosomes of MCF-7 
cells are acidic and hence can trigger the release of Dox from the AuNPs substrate. This acidity is mainly due to the conversion of glucose into lactic acid by cancer cells [26]. Around 70\% of Dox release has been reported at pH 5.3 while only $10 \%$ is released at $\mathrm{pH} 7.4$ in the case of Dox bound to borohydride reduced AuNPs [3].The possibility that electrostatic interactions between AuNPs and Dox are weakened at an acidic $\mathrm{pH}$ which leads to release of Dox has also been suggested. The extracellular tissues of tumors, lysosomes and endosomes are also highly acidic and hence can favor the active release of Dox [27]. The $\mathrm{pH}$ dependent release of Dox from $\mathrm{k}$ and $\mathrm{r}$-AuNPs conjugates may indicate the occurrence of electrostatic interactions and the possibility that it will be released in tumor tissues is high.

\section{Effect of AuNP-Dox conjugates in Zebrafish embryos}

A facile model system for assessing the toxicity and compatibility of nanoparticles is the zebrafish. Since the earlier stages of development (4-96 hpf) are highly sensitive to external agents than the fully developed fish, survival, hatching rate and malformations of the hatched embryos are measured. On continuously monitoring the larvae for a period of $96 \mathrm{hpf}$, no malformation or deformities have been observed after treatment with k-AuNP-Dox and r-AuNP-Dox and they compare well with the untreated group (Figure.3a, b and c). On the other hand, embryos treated with $5 \mu \mathrm{g} / \mathrm{mL}$ of free Dox for a period of 96 hpf showed deformities like bent tail and yolk sac edema (Figure. 3d).

After treatment with different concentrations of k-AuNP-Dox and r-AuNP-Dox, no significant decrease in survival and hatching rate of zebra fish embryos occurred when compared with untreated controls. The survival rates of embryos treated with $60 \mu \mathrm{g} / \mathrm{mL} \mathrm{r}$-AuNPs and their Dox conjugates ( $40 \mu \mathrm{g} / \mathrm{mL})$ after $96 \mathrm{hpf}$ are 83 and $70 \%$ respectively and the results are similar in the presence of the same concentrations k-AuNPs and its Dox conjugate. However in the presence of $5 \mu \mathrm{g} / \mathrm{mL}$ of free Dox, the survival rate of the embryos reduced drastically and reached 38 $\%$ after 96 hpf (Figure. 3e). The hatching rate of embryos after treatment with r-AuNPs-Dox and k-AuNPs-Dox ( $40 \mu \mathrm{g} / \mathrm{mL})$ are found to be 70 and $75 \%$ after $96 \mathrm{hpf}$, whereas delay in hatching process and also comparatively lower hatching rate (58\%) over the same time period has been observed in the group treated with free Dox (Figure. 3f). Using this model, it was previously established that silica nanoparticles are not biocompatible at higher concentrations $(100 \mu \mathrm{g} / \mathrm{mL})$, but at a concentration of about $25 \mu \mathrm{g} / \mathrm{mL}$ the hatching and survival rates were observed to be normal [28].

\section{Uptake of AuNPs-Dox conjugates}

To investigate the cellular internalization of Dox loaded AuNPs conjugates, the treated cells were visualized using CLSM at regular intervals (Figure. 4). The inherent fluorescence property of Dox has been exploited in this study. The red fluorescence of $\mathrm{k}$-AuNP-Dox or r-AuNP-Dox is seen in the cytoplasm of the cells during the first $2 \mathrm{~h}$ (Figure. 4c\&e). After $6 \mathrm{~h}$ of treatment, the nucleus is also found to be stained red (Figure. $4 \mathrm{~d} \& \mathrm{f}$ ). On the other hand, when treated with free Dox, both the cytoplasm and the nucleus are stained red within $2 \mathrm{~h}$ (Figure. $4 \mathrm{a} \& \mathrm{~b}$ ) indicating the slower rate of localization of the AuNP-Dox conjugates when compared to the free Dox. The internalization has also been investigated using flow cytometry (FACS) (Figure. $4 \mathrm{~g} \& \mathrm{~h}$ ). The FACS histograms show that the cells treated with k-AuNPs and r-AuNPs alone do not exhibit fluorescence. The cell associated maximum fluorescence of Dox is observed within $2 \mathrm{~h}$ of treatment when treated with free Dox. However, when cells are treated with equal concentrations of both the AuNPs-Dox conjugates for the same period of time only a low fluorescence signal is noticed. After a treatment period of $6 \mathrm{~h}$, the fluorescence intensity reaches similar values as that of free Dox (Figure. $4 \mathrm{~h}$ ). This indicates the time dependent uptake of the AuNP-Dox conjugates into MCF-7 cells. Our result corroborates well with a previous study in which
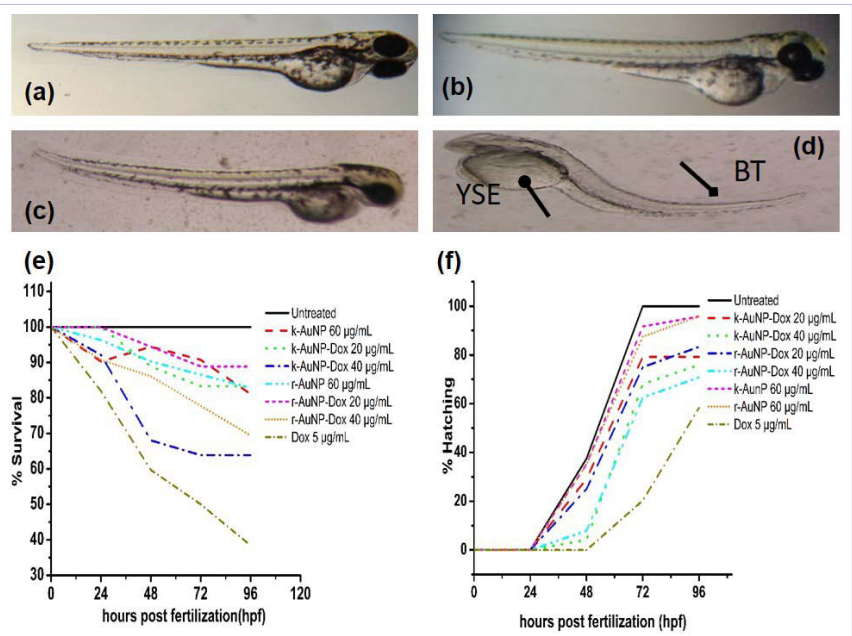

Figure 3: Effect of AuNPs-Dox conjugates on Zebra Fish embryos. Morphological assessment of the larvae ( $96 \mathrm{hpf}$ ) of (a) untreated and treated with (b) k-AuNP- Dox conjugates (c) r-AuNP-Dox (d) free Dox. The arrow indicates $\longrightarrow$ bent tail (BT) and $\longrightarrow$ yolk sac edema (YSE). Three different experiments were performed and the representative images are presented. (e) Survival rates and (f) hatching rates of embryos exposed to AuNPs
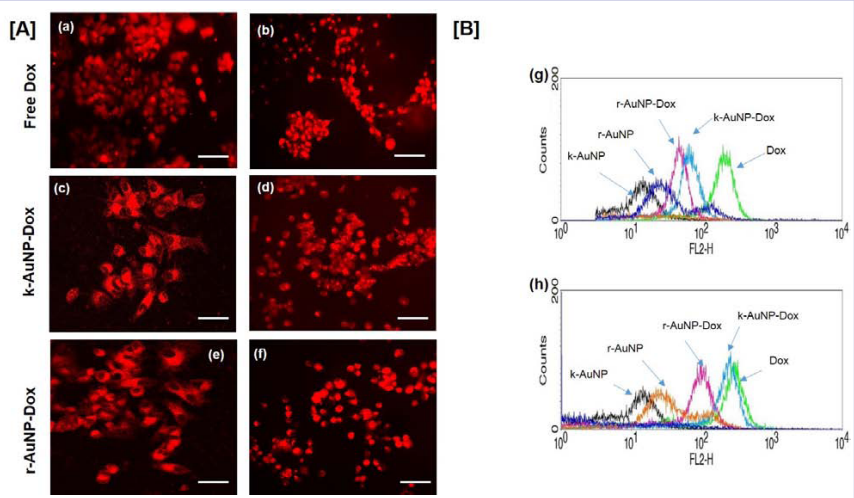

Figure 4: Uptake of Dox loaded AuNPs in MCF-7 cells. [A] Confocal laser scanning micrographs of MCF-7 cells treated with free Dox, k-AuNP-Dox and r-AuNP-Dox conjugates for (a, c \& e) $2 \mathrm{~h}$ and (b, d \& f) $6 \mathrm{~h}$. Scale bar represents $100 \mu \mathrm{m}$. [B] Flow cytometry analysis of MCF-7 cells treated with k-AuNP-Dox and r-AuNP-Dox conjugates after $2 \mathrm{~h} \mathrm{(g)} \mathrm{and} 6 \mathrm{~h}(\mathrm{~h})$ 
a similar time dependent uptake of Dox loaded AuNPs micelles into4T1 cancer cells has been noticed. Free Dox is found to be localized in the nuclear region within $2 \mathrm{~h}$ whereas, the Dox loaded AuNPs are localized in the cytoplasm [29].It is also suggested in another study that free Dox immediately gets transported into the cells via passive diffusion mechanism whereas, Dox loaded polymeric micelles were effectively taken up via endocytosis [30].

The uptake of k-AuNPs and r-AuNPs alone and their corresponding Dox conjugates into MCF-7 cells has also been observed through transmission electron microscopy (TEM) (Figure. 5). The entry of AuNPs and the sub-cellular localization of the AuNPs and AuNP-Dox conjugates in mitochondria is indicated. The mitochondria also show tubular cristae indicating high activity. The mechanism of uptake is by endocytosis and individual nanoparticles are seen without any aggregation. The uptake of AuNPs capped with glucose by MCF-7 cells was also previously reported to be via endocytosis [31].

\section{Cellular viability assay}

After confirming the entry of the drug loaded AuNPs into the cells, cytotoxicity to MCF-7 cells has been evaluated using resazurin cell viability assay. The percent viability of MCF-7 cells has been found to decrease in a concentration and time dependent manner on treatment with both the AuNPs-Dox conjugates. Viability values are found to be $66.5 \pm 5.9,43.2 \pm 4.2$, $39.5 \pm 2.7$ and $28.6 \pm 4.0 \%$ when treated with $10,20,40$ and $60 \mu \mathrm{g} / \mathrm{mL}$ of $\mathrm{r}$-AuNPs-Dox after $24 \mathrm{~h}$. The viability values are similar with k-AuNP-Dox and are $69.4 \pm 5.4,53.7 \pm 2.1,40.8 \pm$ 5.6 and $29.6 \pm 1.4$ in the presence of $10,20,40$ and $60 \mu \mathrm{g} / \mathrm{mL}$ respectively (Figure. 6). Comparison of the $\mathrm{IC}_{50}$ values of $\mathrm{k}$-AuNPDox $(24 \mu \mathrm{g} / \mathrm{mL})$ and r-AuNP-Dox $(15 \mu \mathrm{g} / \mathrm{mL})$ after $24 \mathrm{~h}$ with that of k-AuNPs $(98 \mu \mathrm{g} / \mathrm{mL})$ and r-AuNPs $(71 \mu \mathrm{g} / \mathrm{mL})$ indicates that both k-AuNP-Dox and r-AuNP-Dox conjugates exert higher toxicity to MCF-7 cells when compared to k-AuNPs and r-AuNPs (Figure.S1) [17]. We observe that the k-AuNP-Dox and r-AuNPDox conjugates are 4 and 4.7 times more efficient than the unconjugated k-AuNPs and r-AuNPs respectively in effecting cytotoxicity to MCF-7 cells. The viability values of MCF-7 cells on treatment with 5 and $10 \mu \mathrm{g} / \mathrm{mL}$ of free Dox (corresponding to 20 and $40 \mu \mathrm{g} / \mathrm{mL}$ of the AuNPs concentration) for $24 \mathrm{~h}$ is $70.2 \pm 5.0$ and $57.5 \pm 3.5 \%$ respectively. From this it is also observed that the AuNPs-Dox conjugates are able to reduce the viability of MCF7 cells better than free Dox of the same concentration. Lowering of viability by the AuNP-Dox conjugates can be attributed to the synergistic action of the Dox along with the individual flavonoid. Similar enhanced toxicity of AuNPs-Dox conjugates prepared using aqueous extract of Eclipta alba compared to the AuNPs alone has been reported [32].Our result agrees with the enhanced cytotoxicity exerted by Dox loaded Xanthan gum reduced AuNPs (XGNP) compared to free Dox in A549 cells. The viability after 48 $\mathrm{h}$ is observed to be $50 \%$ when treated with XGNP-Dox conjugate at a corresponding Dox concentration of $1 \mu \mathrm{g} / \mathrm{mL}$ [33]. We also observed a similar percentage of viability when treated with the same concentration and for same period of time in MCF-7 cells. This clearly indicates the effectiveness of Dox conjugated AuNPs in exerting anti-cancer effects.
The viability of HBL-100 cells on treatment with $(60 \mu \mathrm{g} / \mathrm{mL})$ $\mathrm{k}$-AuNP-Dox and r-AuNP-Dox for $24 \mathrm{~h}$ has been observed to be around $72 \%$ while the free Dox treatment drastically reduced the viability of cells to $52.5 \%$ (Figure. 6). This higher reduction in viability of HBL-100 cells by free Dox indicates their toxicity even to normal cells when compared to k-AuNP-Dox and r-AuNP-Dox conjugates. Dox when delivered through AuNPs are observed to exert lower or reduced toxicity to HBL-100 cells while higher cytotoxic effects are noted in MCF-7 cells.

\section{Morphological Assessment}

Morphological assessment has been carried out using the DAPI staining and scanning electron microscopy (SEM). Crescent shaped, brightly stained nucleus indicating condensed chromatin has been observed on treatment with k-AuNP-Dox and r-AuNPDox conjugates (Figure. 7). This shows that $\mathrm{k}$ and r-AuNP-Dox conjugates induce cell death and suggests that the mode of cell death may be apoptosis. Presence of multiple membrane blebs on treatment with a concentration of $40 \mu \mathrm{g} /$ mLof k-AuNPs and r-AuNPs for $48 \mathrm{~h}$ (Figure.8). When the MCF-7 cells were treated with the Dox loaded AuNPs (20 $\mu \mathrm{g} / \mathrm{mL}$ ), presence of multiple membrane blebs have been noticed within $24 \mathrm{~h}$ which clearly indicates that the cell death may occur at an earlier time point.

\section{Cell cycle analysis}

Modulation of various phases of the cell cycle after treatment with the k-AuNP-Dox and r-AuNP-Dox conjugates and free Dox has been assessed. Concentration dependent increase in the percentage of cells in G2/M phase is noticed. On treatment with 10 and $20 \mu \mathrm{g} / \mathrm{mL}$ of $\mathrm{k}$-AuNP-Dox the percent of cells increase from 24.2 to $38.6 \%$. Similar increase from 30.8 to $40.7 \%$ has been observed with 10 and $20 \mu \mathrm{g} / \mathrm{mL}$ of r-AuNP-Dox at $24 \mathrm{~h}$. Free Dox treated MCF-7 cells show $13.4 \%$ of G2/M arrest with $12.2 \%$ of cells in the sub-G1 phase of cell cycle which indicate cell death by apoptosis (Figure.9). The increase in G2/M arrest in the case of Dox conjugates when compared with free Dox indicates
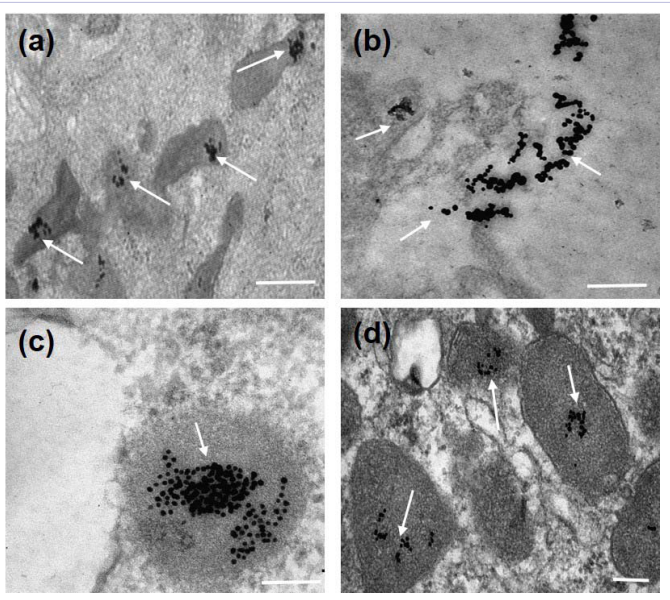

Figure 5: TEM micrographs of MCF-7 cells indicating internalization of AuNP-Dox conjugates after 24 h.(a) k-AuNPs, (b) r-AuNPs, (c) k-AuNPDox and (d) r-AuNP-Dox. Scale bar represents $100 \mathrm{~nm}$ and the arrows indicate the presence of AuNPs 

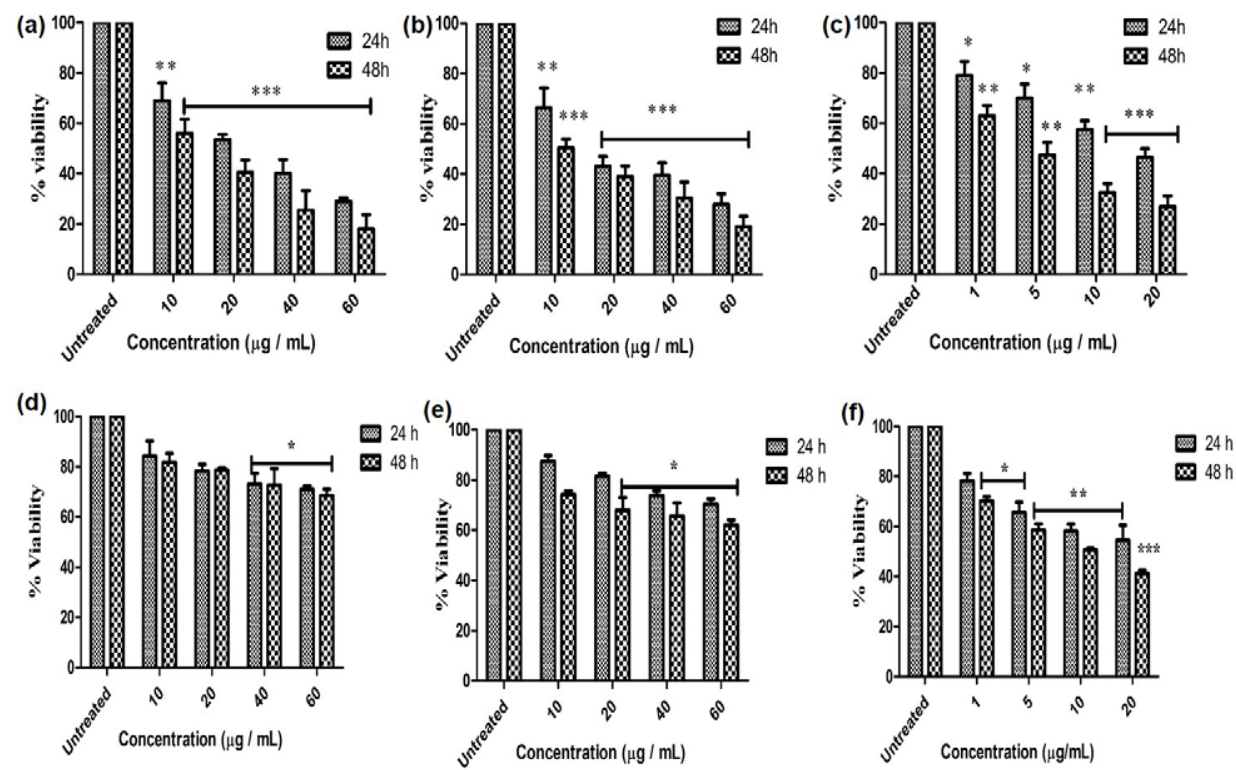

Figure 6: Viability of MCF-7 and HBL-100 cells after treatment with different concentrations of (a, d) k-AuNP-Dox, (b, e) r-AuNP-Dox conjugates and (c, f) equivalent concentrations of free Dox for 24 and $48 \mathrm{~h}$. The experiments are repeated three times and mean \pm SD are represented. P $\leq 0.05$ $*, \mathrm{P} \leq \mathrm{v} 0.01^{* *}$ and $\mathrm{P} \leq 0.001^{* * *}$

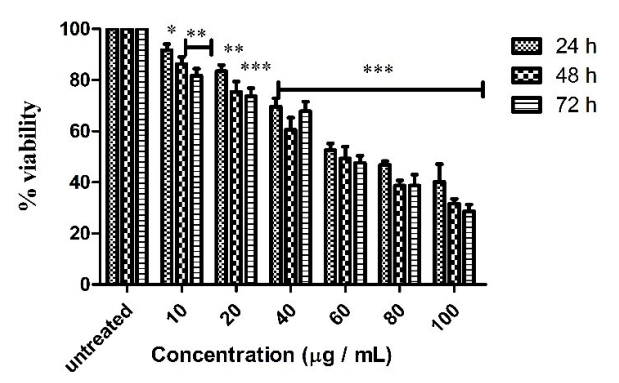

Figure S1: Viability assay of MCF-7 cells after treatment with r-AuNPs alone for different time periods. The experiments are repeated three times and mean $\pm \mathrm{SD}$ are represented. $\mathrm{P} \leq 0.05^{*}, \mathrm{P} \leq 0.01^{* *}$ and $\mathrm{P} \leq$ $0.001^{* * *}$

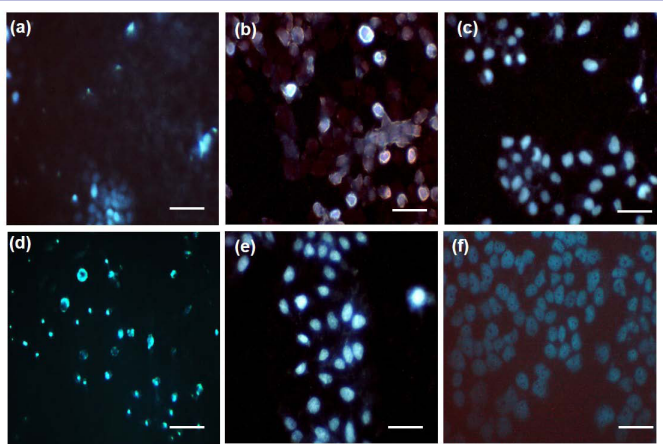

Figure 7: Viability of MCF-7 and HBL-100 cells after treatment with different concentrations of (a, d) k-AuNP-Dox, (b, e) r-AuNP-Dox conjugates and (c, f) equivalent concentrations of free Dox for 24 and 48 h. The experiments are repeated three times and mean \pm SD are represented. $\mathrm{P} \leq 0.05 *, \mathrm{P} \leq \mathrm{v} 0.01 * *$ and $\mathrm{P} \leq 0.001^{* * *}$
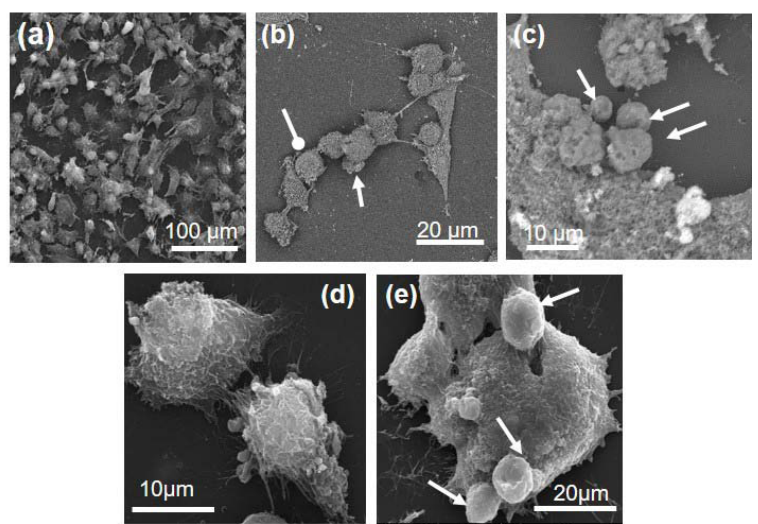

Figure 8: Scanning electron micrographs of MCF-7 cells. (a) untreated, (b) $20 \mu \mathrm{g} / \mathrm{mL}$ of k-AuNP-Dox, (c) $20 \mu \mathrm{g} / \mathrm{mL}$ of r-AuNP-Dox treated cells for $24 \mathrm{~h}$, (d) $40 \mu \mathrm{g} / \mathrm{mL}$ of k-AuNPs and (e) $40 \mu \mathrm{g} / \mathrm{mL}$ of r-AuNPs treated cells for $48 \mathrm{~h}$. Arrows indicate $\longrightarrow$ membrane blebs and $\longrightarrow$ rounded cell

synergistic action of flavonoid, kaempferol or resveratrol along with the Dox. Previously, Docetaxel (anthracycline) loaded lipid nanocapsules have been shown to induce a higher percentage of G2/M arrest in MCF-7 cells than free docetaxel [34].It is also reported earlier that Dox alone or a flavonoid daidzein bring about G2/M arrest in MCF-7 and MDA-MB- 453 cells respectively and induce apoptosis $[35,36]$. Though evidences on the induction of apoptosis by free Dox and also of pure flavonoids have been presented, there are no reports so far on cell cycle arrest by Dox conjugated AuNPs.

\section{DNA Fragmentation}


To detect whether k-AuNPs or r-AuNPs and Dox loaded k-AuNPs or r-AuNPs treatment in MCF-7 cells lead to cleavage of nucleosomal DNA to oligonucleosomal units, DNA fragmentation assay has been performed. The k-AuNPs and r-AuNPs treatments for $48 \mathrm{~h}$ show a ladder like pattern of DNA indicating fragmentation when compared with DNA extracted from untreated intact cells (Figure. S2). In the case of Dox loaded AuNPs, both the conjugates and the free Dox show smear DNA pattern at an earlier time point of $24 \mathrm{~h}$ which is also indicative of cell death by apoptosis (Figure. 10). In the case of HBL-100 cells, fragmented DNA is observed only when treated with free Dox while after treatment with k-AuNP-Dox and r-AuNP-Dox, no fragmentation is noticed and DNA is intact (Figure. S2).Previous reports showed a weak fragmentation and a smear pattern of DNA damage in HeLa and A549 cells upon treatment with AuNPs prepared with leaf extracts of Podophyllum hexandrum and Padina gymnospora leaf broth and it was indicated as apoptotic mode of cell death [37, 38]. But to our knowledge there are no reports on fragmentation of DNA exposed to AuNP-Dox conjugates.

\section{Intracellular ROS generation}

To find out if Dox loaded AuNPs lead to intracellular ROS generation in MCF-7 cells, DCFH-DA has been used as a fluorescent probe. From the fluorescence photomicrographs, bright green fluorescence in MCF-7 cells is seen after treatment for $4 \mathrm{~h}$ with either k-AuNP-Dox or r-AuNP-Dox conjugates when compared to the faint fluorescence in the presence of k-AuNP and r-AuNP alone. This indicates higher ROS production in the presence AuNPDox conjugates (Figure. 11A). This result is supported by FACS analysis.MCF-7 cells on exposure to $10 \mu \mathrm{g} / \mathrm{mL}$ of $\mathrm{k}$ and r-AuNPsDox conjugates exhibit DCF fluorescence of 10 and $20 \%$ after $2 \mathrm{~h}$ indicating ROS production which further increases to 33 and 27 $\%$ respectively after $4 \mathrm{~h}$ (Figure.11B).Higher concentration of 20 $\mu \mathrm{g} / \mathrm{mL}$ induces 23 and $25 \%$ of ROS within $2 \mathrm{~h}$ and eventually reaches 41 and $38 \%$ within $4 \mathrm{~h}$ of treatment with k-AuNP-Dox and r-AuNP-Dox conjugates respectively. After $4 \mathrm{~h}$, both the $\mathrm{k}$ and r-AuNPs ( $60 \mu \mathrm{g} / \mathrm{mL}$ ), without the drug, exhibit low levels of DCF fluorescence of 16 and $14 \%$ respectively. This indicates that the Dox loaded AuNPs conjugates are able to elicit ROS at an earlier time point than the k-AuNPs and r-AuNPs alone. The mode of cell death induced by the AuNPs-Dox conjugates is by apoptosis and is found to be mediated via ROS generation.

\section{Summary}

Surface functionalization of $\mathrm{k}$ and $\mathrm{r}$-AuNPs with the anticancer drug Dox has been achieved. Using zebra fish larvae model system, the Dox loaded AuNPs are found to be non-toxic to the embryos. The release of Dox from AuNPs system is $\mathrm{pH}$ dependent and localization of the drug in the cytoplasm followed by entry into cellular nucleus has been observed. The uptake and enhanced cytotoxicity in cancer cells (MCF-7) has been studied. Both the conjugates induce apoptosis mediated by intracellular ROS generation. The superiority of Dox loaded k and r-AuNPs conjugates over k-AuNPs and r-AuNPs alone has been established.

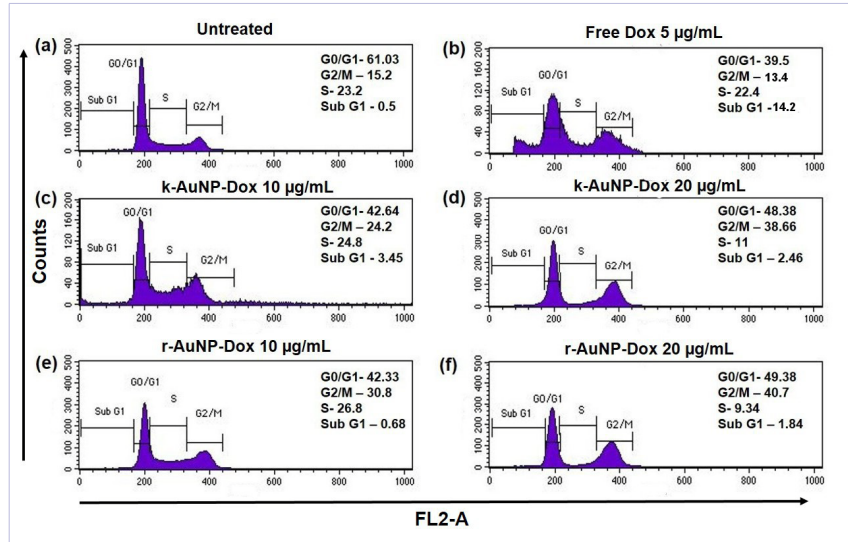

Figure 9: Cell cycle analysis of MCF-7 cells (a) Untreated, (b) $5 \mu \mathrm{g} / \mathrm{mL}$ of free Dox, (c) $10 \mu \mathrm{g} / \mathrm{mL}$ of k-AuNP-Dox, (d) $20 \mu \mathrm{g} / \mathrm{mL}$ of k-AuNPDox, (e) $10 \mu \mathrm{g} / \mathrm{mL}$ of r-AuNP-Dox and (f) $20 \mu \mathrm{g} / \mathrm{mL}$ of r-AuNP-Dox treated cells after $24 \mathrm{~h}$ (a)

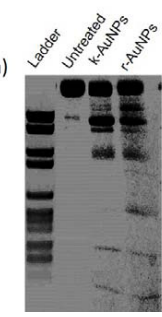

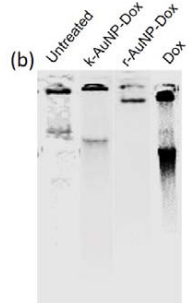

Figure S2: DNA fragmentation analysis of (a) MCF-7 cells treated with $\mathrm{IC}_{50}$ concentrations of k-AuNPs and r-AuNPs alone for $48 \mathrm{~h}$ and (b) HBL100 cells treated with Dox conjugated AuNPs and free Dox for $24 \mathrm{~h}$
Figure 10: Cell cycle analysis of MCF-7 cells (a) Untreated, (b) $5 \mu \mathrm{g} / \mathrm{mL}$ of free Dox, (c) $10 \mu \mathrm{g} / \mathrm{mL}$ of k-AuNP-Dox, (d) $20 \mu \mathrm{g} / \mathrm{mL}$ of k-AuNPDox, (e) $10 \mu \mathrm{g} / \mathrm{mL}$ of r-AuNP-Dox and (f) $20 \mu \mathrm{g} / \mathrm{mL}$ of r-AuNP-Dox treated cells after $24 \mathrm{~h}$

\section{Acknowledgement}

The authors thank the Director, CLRI, Chennai, India for the support provided and CSIR network project, STRAIT (CSC0102) 
[A]

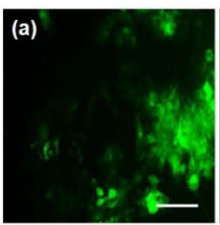

[B]

$2 \mathrm{~h}$
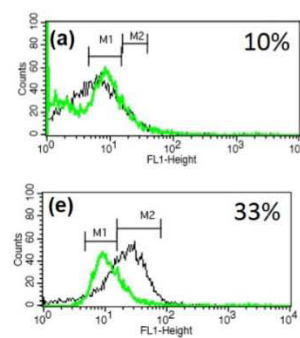

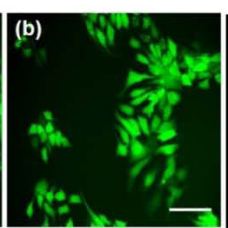

k-AuNP-Dox
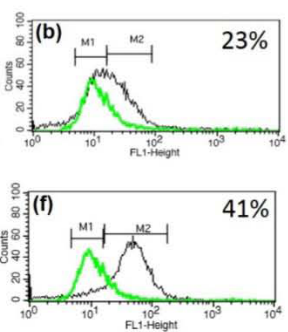
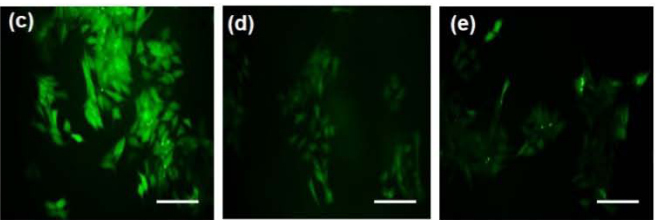

r-AuNP-Dox
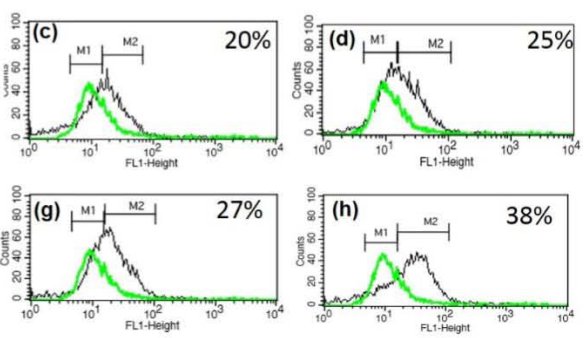

Figure 11: Intracellular ROS production in MCF-7 cells. [A] Fluorescence micrographs of MCF-7 cells on 4 h of treatment with (a) $5 \mu \mathrm{g} / \mathrm{mL}$ of free Dox, (b) $20 \mu \mathrm{g} / \mathrm{mL}$ of k-AuNP-Dox, (c) $20 \mu \mathrm{g} / \mathrm{mL}$ of r-AuNP-Dox, (d) $40 \mu \mathrm{g} / \mathrm{mL}$ of k-AuNPs and (e) $40 \mu \mathrm{g} / \mathrm{mL}$ of r-AuNPs. Scale represents 100 $\mu \mathrm{m}$. [B] FACS analysis of MCF-7 cells treated with k-AuNP-Dox (a, e) $10 \mu \mathrm{g} / \mathrm{mL}$, (b, f) $20 \mu \mathrm{g} / \mathrm{mL}$ and r-AuNP-Dox (c, g) $10 \mu \mathrm{g} / \mathrm{mL}(\mathrm{d}, \mathrm{h}) 20 \mu \mathrm{g} / \mathrm{mL}$

for financial assistance. One of the authors (S.R.B) wishes to thank the DST INSPIRE, New Delhi, India, for the Senior Research Fellowship.

\section{References}

1. Crozier JA, Swaika A, Moreno-Aspitia A. World. J. Clin. Oncol 2014;5(3):529-538. doi: 10.5306/wjco.v5.i3.529.

2. Dixit V, Van den Bossche J, Sherman DM, Thompson DH, Andres RP. Bioconjugate Chem. 2006;17(3):603-609. DOI: 10.1021/bc050335b

3. Aryal S, Grailer JJ, Pilla S, Steeber DA and Gong S. J. Mater. Chem., 2009; 19, 787.

4. Peer D, Karp JM, Hong S, Farokhzad OC, Margalit R, Langer R.. Nat. Nanotechnol. 2007;2(12):751-760. doi: 10.1038/nnano.2007.387

5. Hu FQ, Liu LN, Du YZand Yuan H, Biomaterials. 2009; 30, 6955.

6. Harris L, Batist G, Belt R, Rovira D, Navari R, Azarnia N, et al. 2002;94(1):25-36.

7. Wang Y, Cao X, Guo R, Shen M, Zhang M, Zhu M and Shi X. Polym. Chem. $2011 ; 2,1754$.

8. Yang J, Lee CH, Park J, Seo S, Lim EK, Song YJ, Suh JS, Yoon HG, Huh YM and Haam S. J. Mater. Chem. 2007; 17, 2695.

9. Liu Z, Sun X, Nakayama-Ratchford N, Dai H. ACS Nano. 2007;1(1):5056. doi: 10.1021/nn700040t.

10. Jain TK, Morales MA, Sahoo SK, Leslie-Pelecky DL, Labhasetwar V. Mol. Pharm. 2005;2(3):194-205.

11. Venkatesan R, Pichaimani A, Hari K, Balasubramanian PK, Kulandaivel J and Premkumar K. J. Mater. Chem. B. 2013;1:1010-1018. DOI: 10.1039/C2TB00078D

12. Park C, Youn H, Kim H, Noh T, Kook YH, Oh ET, Park HJ and Kim C. J. Mater. Chem. 2009; 19, 2310.

13. Heo DN, Yang DH, Moon HJ, Lee JB, Bae MS, Lee SC, et al. Biomaterials. 2012;33(3):856-866. doi: 10.1016/j.biomaterials.2011.09.064.

14. Doane T and Burda C. Adv. Drug. Deliv. Rev. 2013;65(5):607-21. doi: 10.1016/j.addr.2012.05.012.
15. Kumar SA, Peter YA and Nadeau JL. Nanotechnology. 2008;19(49):495101. doi: 10.1088/0957-4484/19/49/495101.

16. Pandey S, Oza G, Mewada A, Shah R, Thakur M and Sharon M. J. Mater. Chem. 2013;9: 1361-1370. DOI: 10.1039/C2TB00168C.

17. Bhuvanasree SR, Kondath S, Anantnarayanan R and Rama Rajaram. Process Biochemistry, 2015; 50, 1966.

18. Mirza AZ and Shamshad H. Eur. J. Phys. Chem. 2011;46(5):1857-1860. doi: 10.1016/j.ejmech.2011.02.048.

19. EU Directive, 2010/63/EU, 236, 33.

20.0'Brien J, Wilson I, Orton T and Pognan F. Eur. J. Biochem. 2000;267(17):5421-5426.

21. Herrmann M, Lorenz HM, Voll R, Grunke M, Woith W and Kalden JR. Nucleic Acids Res. 1994;22(24):5506-5507.

22. Li N, Chen Y, Zhang YM, Yang Y, Su Y, Chen JT, Liu Y. Scientific Reports. $2014 ; 4,4164$.

23. Wang F, Wang YC, Dou S, Xiong MH, Sun TM and Wang J. ACS Nano. 2011;5(5):3679-3692. doi: 10.1021/nn200007z.

24. Dhar S, Reddy EM, Shiras A, Pokharkar V and Prasad BLV. Chem. Eur. J. 2008;14(33):10244-10250. doi: 10.1002/chem.200801093.

25. Madhusudhan A, Reddy GB, Venkatesham M, Veerabhadram G, Anil Kumar D, Natarajan S, et al. Int. J. Mol. Sci. 2014;15(5):8216-8234. doi:10.3390/ijms15058216

26. Altan N, Chen Y, Schindler $M$ and Simon SM. J. Exp. Med. 1998;187(10):1583-1598.

27.You J, Zhang G and Li C, ACS Nano. 2010;4(2):1033-1041. doi: 10.1021/nn901181c.

28. Duan J, Yu Y, Shi H, Tian L, Guo C, Huang P, et al. PLOS one. 2013;8(9):e74606. doi: 10.1371/journal.pone.0074606.

29. Prabaharan M, Grailer JJ, Pilla S, Steeber DA and Gong S. Biomaterials, 2009;30(30):6065-6075. doi: 10.1016/j.biomaterials.2009.07.048 
30. Yoo HS and Park JG. J. Control. Release. 2001;70(1-2):63-70.

31. Song K, Xu P, Meng Y, Geng F, Li J, Li Z, etal. Int. J. Oncol. 2013;42(2):597608. doi: 10.3892/ijo.2012.1721.

32. Mukherjee S, Sushma V, Patra S, Barui AK, Bhadra MP, Sreedhar B, et al. Nanotechnology. 2012;23(45):455103. doi: 10.1088/0957$4484 / 23 / 45 / 455103$.

33. Pooja D, Panyaram S, Kulhari H, Rachamalla SS and Sistla R.Carbohydr. Polym. 2014;110:1-9. doi: 10.1016/j.carbpol.2014.03.041.

34. Sanchez-Moreno P, Boulaiz H, Ortega-Vinuesa JL, Peula-GarciaJM and Aranega A. Int. J. 2012;13(4):4906-4919. doi: 10.3390/ijms13044906.
35. Meiyanto E, Hermawan A, Junedi S, Fitriasari A and Susidarti RA. Int. J. Phytomed. 2011; 3, 129.

36. Choi EJ and Kim GH. Phytomedicine. 2008;15(9):683-690. doi: 10.1016/j.phymed.2008.04.006.

37. Jeyaraj M, Arun R, Sathishkumar G, Mubarak Ali D, Rajesh M, Sivanandhan G, Kapildev G, Manickavasagam M, Thajuddin N and Ganapathi A. Mater. Res. Bull. 2014; 52, 15.

38. Singh M, Kumar M, Manikandan S, Chandrasekaran N, Mukherjee A and Kumaraguru AK, J.Nanomed. Nanotechnol. 2014;S5:009. doi:10.4172/2157-7439.S5-009 